\title{
A IMPORTÂNCIA DA NOTIFICAÇÃO DOS AGRAVOS EM SAÚDE DO TRABALHADOR
}

\author{
Lílian Salomão Elias ${ }^{1}$
}

\section{RESUMO}

O processo de atenção integral à saúde dos trabalhadores, deve estar presente em todos os serviços de saúde, buscando construir ambientes e processos de trabalho saudáveis tendo como principal ferramenta as notificações que tornam o atendimento mais efetivo. A atuação de vigilância em saúde do trabalhador deve ser contínua, sistemática, mantendo as informações que apontem os problemas de saúde e os fatores de risco relacionados ao processo de trabalho, propondo ações de planejamento de ações que evitem agravos. Esse estudo aponta como problema o desconhecimento e a falta de notificação dos agravos ocorridos com os trabalhadores e tem como objetivos despertar nos profissionais o interesse em notificar os acidentes relacionados ao trabalho, alimentando o sistema e possibilitando a criação de políticas públicas voltadas para a prevenção desses agravos. O método de pesquisa utilizado foi a revisão bibliográfica por meio da seleção de artigos científicos provenientes das bases de dados Scielo, políticas nacionais e leis, publicações do ministério da saúde com ligação ao tema. A realização deste trabalho possibilitou o conhecimento sobre a realidade das notificações referentes à saúde de trabalhador e destacou a importância da construção de ambientes e processos de trabalho saudáveis. Conclui-se, portanto, que, para que ocorra melhoria das notificações de atendimentos relacionados ao trabalho, os profissionais devem trabalhar a humanização no atendimento aos acidentados além de receberem o apoio dos gestores em situações que requerem maior complexidade.

Palavras-chave: Saúde. Trabalhador. Agravos. Notificação. 


\section{SUMMARY}

The process of integral care to workers' health should be present in all health services, seeking to build healthy work environments and processes with notifications that make care more effective. The action of health surveillance of workers should be continuous, systematic, maintaining the information that shows health problems and risk factors related to the work process, proposing actions to plan actions that avoid injuries. This study points out as a problem the lack of knowledge and the lack of notification of injuries that occurred with workers and aims to awaken in professionals the interest in notifying work-related accidents, feeding the system and enabling the creation of public policies aimed at the prevention of these injuries. The research method used was the literature review through the selection of scientific articles from the Scielo databases, national policies and laws, publications of the Ministry of Health with connection to the theme. The accomplishment of this work allowed the knowledge about the reality of notifications related to workers' health and highlighted the importance of building healthy work environments and processes. It is concluded, therefore, that, in order to improve notifications of work-related care, professionals must work on humanization in the care of injured victims in addition to receiving the support of managers in situations that require greater complexity.

Keywords: health, worker, injuries, notification 


\section{1- INTRODUÇÃO}

A notificação de acidente de trabalho (AT) é hoje um importante instrumento para a saúde pública, pois a partir dela se tem real conhecimento sobre a situação dos trabalhadores atendidos pelo Sistema Único de Saúde (SUS). No Brasil, até 1988, a Saúde era apenas um benefício previdenciário (restrito aos contribuintes) ou um serviço comprado na forma de assistência médica ou, por fỉm, uma ação de misericórdia oferecida aos que não tinham acesso à previdência e nem recursos para pagar a assistência privada, prestada por hospitais filantrópicos, como as Santas Casas. Desse modo, a atenção à saúde era, rigorosamente, um serviço oferecido e regulado pelo mercado ou pela Previdência Social, por meio de uma política de Estado compensatória voltada aos trabalhadores contribuintes, formalmente inseridos no mercado de trabalho. (MINISTÉRIO DA SAÚDE, 2004) Naquela época, as ações de caráter mais coletivo ou as "ações de saúde pública" eram executadas pelo Ministério da Saúde e completamente dissociadas da atenção individual. Essas ações resumiam-se em campanhas e programas predominantemente de caráter preventivista, como as campanhas de vacinação e os programas verticais sobre doenças endêmicas, como tuberculose, hanseníase, doença de Chagas, malária, entre outras. (MINISTÉRIO DA SAÚDE, 2004). Tal modelo, que dissociava as ações individuais das ações coletivas e excluía grande parte da população da atenção à saúde, aliado aos níveis de desigualdade de distribuição da riqueza do país, contribuía incisivamente para perpetuar péssimas condições de saúde e qualidade de vida aos cidadãos. A partir de meados dos anos 70 e durante toda a década de 80 , o recrudescimento dos movimentos sociais levou o Brasil ao seu processo de redemocratização. Nesse contexto surge o Movimento de Reforma Sanitária, propondo uma nova concepção de Saúde Pública para o conjunto da sociedade brasileira, incluindo a Saúde do Trabalhador. Dessa forma, a Saúde do Trabalhador reflete uma resposta institucional aos movimentos sociais que, entre a metade dos anos 70 e os anos 90, reivindicavam que as questões de saúde relacionadas ao trabalho fizessem parte do direito universal à saúde, incluídas no escopo da Saúde Pública. (MINISTÉRIO DA SAÚDE, 2004).

As ações em Saúde do Trabalhador, no âmbito do SUS, têm se desenvolvido de forma isolada e fragmentada das demais ações de saúde. Estas ações atualmente são desenvolvidas de modo desigual nos estados e municípios. Tal atraso no cumprimento constitucional para as ações em Saúde do Trabalhador no SUS, tem se refletido em alguns indicadores de mortalidade e gravidade elevados. O direito à saúde e à vida, passa pela transformação do processo de produção, que de fonte de agravos e de morte deve ser um fator de proteção e de promoção da vida. Neste contexto, o Sistema Único de Saúde tem um papel fundamental, sendo racional e adequado que a rede de serviços públicos de saúde se qualifique e estruture para atender as demandas de saúde do trabalhador de forma integral. Desta forma, demonstra-se a necessidade da formulação de uma Política Nacional de Saúde do Trabalhador para o SUS que atenda esta demanda. Política está entendida como o instrumento orientador da atuação do setor saúde no campo da saúde dos trabalhadores, com o objetivo de promover e proteger a saúde dos trabalhadores por meio de ações de promoção, vigilância e assistência; além de explicitar as atribuições do setor saúde no que se refere às questões de Saúde do Trabalhador de modo a dar visibilidade à questão e viabilizar a pactuação intra e inter-setorial e fomentar a participação e o controle social. 


\section{2 - MÉTODOS}

Foi realizado um estudo de abordagem qualitativa, do tipo bibliográfico, resumindo a literatura teórica e fornecendo compreensão ampla sobre um dado fenômeno. As buscas por material bibliográfico foram realizadas utilizando como base de dados a plataforma Scientific Electronic Library Online (Scielo), Instituições Públicas e Google Acadêmico, em língua portuguesa e foram excluídos trabalhos cujo foco seja a gestão de enfermagem em outros setores, que não a hospitalar. Foram separados 26 artigos, dos quais 10 foram excluídos por fugirem do tema.

\section{2- DESENVOLVIMENTO}

\section{1 - A Saúde do Trabalhador}

A Lei Orgânica da Saúde (Lei Federal 8080/90), em seu artigo $6^{\circ}$, parágrafo $3^{\circ}$, regulamenta os dispositivos constitucionais sobre Saúde do Trabalhador, da seguinte forma: "Entende-se por saúde do trabalhador, para fins desta lei, um conjunto de atividades que se destina, através das ações de vigilância epidemiológica e vigilância sanitária, à promoção e proteção da saúde dos trabalhadores, assim como visa à recuperação e reabilitação da saúde dos trabalhadores submetidos aos riscos e agravos advindos das condições de trabalho, abrangendo:

I - assistência ao trabalhador vítima de acidentes de trabalho ou portador de doença profissional e do trabalho;

II - participação, no âmbito de competência do Sistema Único de Saúde (SUS), em estudos, pesquisas, avaliação e controle dos riscos e agravos potenciais à saúde existentes no processo de trabalho;

III - participação, no âmbito de competência do Sistema Único de Saúde (SUS), da normatização, fiscalização e controle das condições de produção, extração, armazenamento, transporte, distribuição e manuseio de substâncias, de produtos, de máquinas e de equipamentos que apresentam riscos à saúde do trabalhador;

IV - avaliação do impacto que as tecnologias provocam à saúde;

V - informação ao trabalhador e à sua respectiva entidade sindical e às empresas sobre os riscos de acidentes de trabalho, doença profissional e do trabalho, bem como os resultados de fiscalizações, avaliações ambientais e exames de saúde, de admissão, periódicos e de demissão, respeitados os preceitos da ética profissional;

VI - participação na normatização, fiscalização e controle dos serviços de saúde do trabalhador nas instituições e empresas públicas e privadas; 
VII - revisão periódica da listagem oficial de doenças originadas no processo de trabalho, tendo na sua elaboração a colaboração das entidades sindicais;

VIII - a garantia ao sindicato dos trabalhadores de requerer ao órgão competente a interdição de máquina, de setor de serviço ou de todo ambiente de trabalho, quando houver exposição a risco iminente para a vida ou saúde dos trabalhadores.".

Dessa forma, a configuração da Saúde do Trabalhador se dá diretamente no âmbito do direito à saúde, previsto como competência do SUS. Devido à abrangência de seu campo de ação, apresenta caráter intra-setorial (envolvendo todos os níveis de atenção e esferas de governo do SUS) e inter-setorial (envolvendo a Previdência Social, Trabalho, Meio Ambiente, Justiça, educação e demais setores relacionados com as políticas de desenvolvimento), exigindo uma abordagem interdisciplinar e com a gestão participativa dos trabalhadores. (BRASIL, 1990)

\section{2 - Ações em Saúde do Trabalhador}

As ações de Saúde do Trabalhador compreendem a assistência aos agravos, a vigilância dos ambientes e condições de trabalho (vigilância sanitária), da situação de saúde dos trabalhadores (vigilância epidemiológica) e da situação ambiental (vigilância ambiental); a produção, coleta, sistematização, análise e divulgação das informações de saúde, a produção de conhecimento e as atividades educativas, todas elas desenvolvidos sob o controle da sociedade organizada. A partir das ações assistenciais são identificados os "casos" ou situações de adoecimento relacionadas ao trabalho, que são notificados ao Sistema de Informação, desencadeando procedimentos de vigilância da saúde. ( MANUAL DE GESTÃO E GERENCIAMENTO, 2006) As ações de vigilância dos ambientes e condições de trabalho, vigilância epidemiológica de agravos e da vigilância ambiental também geram informação e identificam "casos de doentes ou de suspeitos" que são encaminhados à rede de serviços sentinela, para diagnóstico e, se necessário, para tratamento e reabilitação. Fecha-se, dessa forma, o ciclo da atenção integral à saúde dos trabalhadores, que inclui ainda, procedimentos de promoção da saúde definidos e implementados no âmbito do sistema de saúde e fora dele, pelo setor Trabalho, Previdência Social, Meio Ambiente e outros setores de governo responsáveis pelas políticas de desenvolvimento econômico e social. A escassez e inadequação das informações sobre a real situação de saúde dos trabalhadores dificultam a definição de prioridades para o planejamento e intervenções em saúde, além de omitir à sociedade instrumentos importantes para a melhoria das condições de trabalho.( MANUAL DE GESTÃO E GERENCIAMENTO, 2006) A Vigilância em Saúde do Trabalhador (VISAT) é um componente do Sistema Nacional de Vigilância em Saúde, como definido na Portaria GM/MS n 3252 de dezembro de 2009, que visa à promoção da saúde e à redução da morbimortalidade da população trabalhadora, por meio da integração de ações que intervenham nos agravos e seus determinantes decorrentes dos modelos de desenvolvimento e processo produtivos. A VISAT é estruturante e essencial ao modelo de Atenção Integral em Saúde do Trabalhador. Constitui-se de saberes e práticas sanitárias, articulados intra e inter setorialmente. A especificidade de seu campo de ação é definida por ter como objeto a relação da saúde com o ambiente e os processos de trabalho, realizada com a participação e o saber dos trabalhadores em todas as suas etapas. (RENAST, ONLINE) A VISAT pauta-se nos princípios do Sistema Único de Saúde, em consonância com a Promoção da Saúde e o Sistema Nacional de Vigilância 
em Saúde, mantendo estreita integração com as demais Vigilâncias - sobretudo com a Sanitária, Epidemiológica e Saúde Ambiental - e as redes assistenciais.

A essência da ação de vigilância em saúde do trabalhador é desenvolvida por análises de documentos, entrevistas com trabalhadores e observação direta do processo de trabalho. Corresponde ao modo de olhar do sanitarista para o trabalho na tentativa de destacar seus impactos a saúde e ao meio ambiente. É exercida por uma equipe de Vigilância em Saúde do Trabalhador, não devendo ser realizada de forma individual. .(RENAST, ONLINE) A Política do Ministério da Saúde para a Informação e Informática inclui a Saúde do Trabalhador entre as ações de saúde coletiva, ao lado das ações de Vigilância Sanitária e Vigilância Ambiental e destaca os ganhos de eficiência e qualidade a serem obtidos pelo uso da informática. .( MANUAL DE GESTÃO E GERENCIAMENTO, 2006) A Política tem como propósito: "promover o uso inovador, criativo e transformador da tecnologia da informação, para melhorar os processos de trabalho em saúde, resultando em um Sistema Nacional de Informação em Saúde articulado, que produza informações para a gestão, a prática profissional, a geração de conhecimentos e o controle social, garantindo ganhos de eficiência e qualidade mensuráveis através da ampliação de acesso, equidade, integralidade e humanização dos serviços".

Assim, a informação em Saúde do Trabalhador deve ser construída como parte do Sistema Nacional de Informação em Saúde, em sintonia com os novos paradigmas e as principais diretrizes desta Política.( MANUAL DE GESTÃO E GERENCIAMENTO, 2006)As ações e informações oriundas do nível local deverão alimentar os demais níveis do Sistema de Informação de Saúde, favorecendo, nos âmbitos estadual e federal ações de prevenção e intervenção sobre os problemas de maior abrangência e complexidade. As informações geradas devem ser fidedignas e socializadas para orientar as ações em Saúde do Trabalhador. A análise das informações obtidas, a partir da vigilância e do acompanhamento da situação de saúde dos trabalhadores, deverá ser disponibilizada, em tempo oportuno, de modo a subsidiar o planejamento e a execução de ações de saúde. Devem ainda permitir a ação conjunta e articulada entre os vários setores, órgãos, instituições e atores envolvidos, favorecendo o processo de controle social. Para isso, será igualmente importante o estabelecimento de mecanismos permanentes de comunicação com a sociedade e, em particular, com os trabalhadores, garantindo ampla difusão dessas informações, incluindo sua disponibilidade via Internet e outros meios. (MANUAL DE GESTÃO E GERENCIAMENTO, 2006)

\section{3 - Participação da Comunidade na Gestão das Ações em Saúde do Trabalhador}

É importante considerar que as ações de saúde deverão ser desenvolvidas em conjunto com representantes da sociedade civil; os trabalhadores e suas organizações, sindicais e de locais de trabalho, outras formas de representação social que congreguem os trabalhadores de setores da economia informal, de produção agrícola, autônomos e outros; dos empregadores; grupos sociais interessados no desenvolvimento sustentável, como os movimentos ambientalistas, com vistas à identificação de soluções e compromissos que favoreçam a promoção e a proteção da saúde dos trabalhadores. (MINISTÉRIO DA SAÚDE, 2004) O controle social, uma premissa básica do SUS ganha relevância na Política de Saúde do Trabalhador, uma vez que, a participação dos trabalhadores é essencial para a identificação dos fatores de risco presentes nos processos de trabalho, das repercussões sobre o processo saúdedoença e das transformações das condições geradoras de acidentes e doença. Na atualidade, as mudanças nos processos produtivos e na estrutura sindical têm obrigado a busca de alternativas para a representação dos trabalhadores nos organismos colegiados. $\mathrm{O}$ crescimento das relações 
informais e precárias de trabalho fragiliza a prática sindical e exige a criação de novas modalidades de representação dos trabalhadores. (MINISTÉRIO DA SAÚDE, 2004)

\section{4- A SAÚDE DO TRABALHADOR NO MEIO AMBIENTE DE TRABALHO E A IMPORTÂNCIA DA NOTIFICAÇÃO PARA AS POLÍTICAS DE PROMOÇÃO E PROTEÇÃO À SAÚDE DO TRABALHADOR}

\section{1- As inter-relações entre a Saúde do Trabalhador e a Saúde Ambiental}

A expansão industrial, ocorrida após a Segunda Guerra Mundial resultou em mudanças no mundo do trabalho e na ampliação das doenças relacionadas ao trabalho. Incorporou aspectos do ambiente de trabalho, que também se tornaram campo de intervenção na área, superando assim o viés eminentemente clínico da medicina e incorporando outros saberes tais como da engenharia, da psicopatologia, da psicodinâmica e da sociologia do trabalho.

No ambiente de trabalho, as novas tecnologias e as relações de trabalho apresentam riscos tecnológicos, de natureza física, química, biológica, mecânica, ergonômica e psíquica que podem causar acidentes do trabalho e doenças relacionadas ao trabalho, cujos impactos são gerados pelos processos de produção e consumo que se manifestam sob a forma de acidentes que causam mortes, lesões corporais, intoxicações, alergias respiratórias, cânceres e malformações congênitas. (SERVO, 2005)

A abordagem integrada das inter-relações entre as questões de saúde do trabalhador e saúde ambiental representa na atualidade, um grande desafio para o SUS, uma vez que, em muitos casos, a degradação ambiental originada no processo de produção, armazenagem, expedição, distribuição e comercialização, expressas na poluição do ar, solo, água superficial e subterrânea, causam danos à saúde dos trabalhadores e da população do entorno. (MINISTÉRIO DA SAÚDE, 2004)

Neste sentido, a Epidemiologia Social propicia o encontro entre os campos disciplinares da saúde ambiental e da saúde do trabalhador, sinalizando novas perspectivas para o desvelamento de agravos à saúde, decorrentes das modificações nas formas e nos processos de trabalho e para o planejamento, execução e avaliação das ações de saúde e formulação de novas políticas para o setor. (SERVO, 2005)

Nessa perspectiva, as ações de saúde passam a ser orientadas pela necessidade de identificar os fatores de risco presentes nos processos de trabalho, buscando suprimir ou controlar a exposição, por meio de ações de vigilância em saúde, configurando uma atuação multiprofissional, interdisciplinar e intersetorial capaz de contemplar a complexidade das relações produção-consumo-ambiente e saúde. (MINISTÉRIO DA SAÚDE, 2004) A Vigilância à Saúde do Trabalhador deve manter-se como uma atuação contínua e sistemática, no sentido de detectar, conhecer, pesquisar e analisar os fatores determinantes e condicionantes dos agravos à saúde relacionados aos processos e ambientes de trabalho, em seus aspectos tecnológico, social, organizacional e epidemiológico, com o objetivo de planejar, executar e avaliar intervenções sobre esses aspectos, de forma a eliminá-los ou controlá-los. (TAMASSIRO, 2009) A construção de ambientes e processos de trabalho saudáveis; o 
fortalecimento da vigilância de ambientes, processos e agravos relacionados ao trabalho e a assistência integral à saúde caracterizam a atenção integral à saúde. Pressupõem a adequação e ampliação da capacidade institucional de modo a permitir que este conjunto de ações seja desenvolvido com competência e resolutividade pela rede de serviços de saúde, em ação coordenada de diversos setores do governo e da sociedade civil. A articulação intra - setorial envolve todas as instâncias do SUS. No Ministério da Saúde, a Área Técnica de Saúde do Trabalhador é o ponto focal dessa articulação e abrange, no campo da assistência, a Atenção Básica, a Média e Alta Complexidade e os programas e ações direcionadas a grupos populacionais específicos, entre eles: as mulheres, os idosos; criança e adolescente; portadores de necessidades especiais. (DIAS \& HOEFEL)

No Brasil anualmente, centenas de trabalhadores sofrem com condições de trabalho precárias sem a assistência adequada à prevenção. Muitas vezes o ambiente de trabalho é completamente impróprio, e o trabalhador por desconhecer ou não identificar determinadas situações de risco, ou apenas para garantir sua subsistência, sofre acidentes do trabalho ou doenças ocupacionais. O planejamento das ações embasadas é essencial para o sucesso da assistência prestada ao trabalhador. Para a correta promoção da saúde, a Equipe de Atenção Básica e o profissional enfermeiro, precisam adequar-se aos princípios do SUS e cumprir as ações propostas para o nível local de saúde obedecendo às atribuições gerais e específicas preconizadas pelo Ministério da Saúde. Através desta revisão, constata-se que o papel dos profissionais de saúde é essencial na participação das ações a serem desenvolvidas em benefício ao trabalhador. (MINISTÉRIO DA SAÚDE, 2004)

\section{2 - A atenção integral à saúde do trabalhador}

A organização de uma atenção diferenciada à saúde dos trabalhadores surge, no mundo ocidental, no século 18, na Inglaterra, com a Revolução Industrial. Pressionados pelos prejuízos econômicos, decorrentes dos altos índices de acidentes e adoecimento determinados pelas péssimas condições de vida e trabalho e pelas reivindicações dos trabalhadores por mudanças, industriais da época passaram a contratar médicos, atribuindo-lhes a responsabilidade de "cuidar" da saúde dos trabalhadores. (SANTANA)

A maioria dos estudos sobre acidentes de trabalho provém de informações contidas nas Comunicações de Acidentes de Trabalho - CAT e das Declarações de Óbito. Porém, estes sistemas de informações são alimentados apenas com dados referentes ao trabalhador formal, havendo mesmo assim uma acentuada subnotificação das informações (SERVO, 2000).

Um modelo de atenção integral à saúde dos trabalhadores implica em qualificar as práticas de saúde, envolvendo o atendimento dos acidentados do trabalho, dos trabalhadores doentes, das urgências e emergências, às ações de promoção e proteção da saúde e de vigilância, orientadas por critério epidemiológico. Para que ocorra de modo efetivo, exige abordagem interdisciplinar e a utilização de instrumentos, saberes, tecnologias originadas de diferentes áreas do conhecimento, colocados a serviço das necessidades dos trabalhadores. (MINISTÉRIO DA SAÚDE, 2004)

O processo de construção da Rede Nacional de Atenção Integral à Saúde do Trabalhador RENAST, pela Portaria MS $\mathrm{N}^{\circ} 1679 / 02$ no SUS, representou o aprofundamento da institucionalização e do fortalecimento da saúde do trabalhador, no âmbito do SUS, em nosso país, reunindo as condições para o estabelecimento de uma política de estado e os meios para 
sua execução, articulando a concepção de uma rede nacional, cujo eixo integrador é a rede regionalizada de Centros de Referencia em Saúde do Trabalhador - CRSTs, localizados em cada uma das capitais, regiões metropolitanas e municípios sede de pólos de assistência, das regiões e micro-regiões de saúde, com a atribuição de dar suporte técnico e científico às intervenções do SUS no campo da saúde do trabalhador, integradas, no âmbito de uma determinada região, com a ação de outros órgãos públicos.

A institucionalização viabilizou uma estratégia de disseminação das ações em saúde do trabalhador em toda rede de serviços do SUS - Unidades Básicas de Saúde, Ambulatórios, Pronto-Socorro e Hospitais - distribuídos em todos os 5.561 municípios brasileiros. (MINISTÉRIO DA SAÚDE, 2004)

\section{5- CONSIDERAÇÕES FINAIS}

A dinâmica da produção, as condições de trabalho e o modo de vida continuam sendo fontes importantes na compreensão do processo de saúde, adoecimento e morte dos trabalhadores. Mesmo os acidentes que ocorrem pelo descuido do trabalhador podem ser condicionados por diferentes determinantes: o cansaço pelas horas extras, estafa crônica, horas não dormidas, alimentação e transporte deficientes, precárias condições ambientais, más condições de vida e de trabalho, dentre outras. Essas devem ser orientadas como situações de risco para o trabalhador e instruídas pelo profissional de saúde. (SERVO,2005)

A dificuldade dos profissionais de saúde em reconhecer os acidentados de trabalho evidencia que o acidente de trabalho não é percebido como problema de saúde pública, dificultando a aproximação ao entendimento de que o acidente de trabalho é uma forma de violência e que necessita para a promoção e proteção da saúde dos trabalhadores da sua notificação e da produção de conhecimentos que realizem a articulação destes com o campo de estudos sobre a violência. $\mathrm{O}$ desconhecimento sobre acidente de trabalho pelo trabalhador também reafirma o desconhecimento dos riscos existentes no meio ambiente de trabalho e a necessidade de capacitação destes quanto aos riscos do mundo do trabalho e a as medidas necessárias para a promoção e proteção à saúde do trabalhador.

No cenário de atendimento ao acidentado nas unidades de saúde e emergência, identifica-se problemas de organização do serviço, comuns na maioria dos municípios.

Alguns pontos precisam ser analisados e melhorados, tais como: Identificação correta e completa dos atendimentos por causa externa; Orientação aos profissionais de saúde sobre o atendimento ao acidentado do trabalho; Preparação do profissional de saúde, afim de que o mesmo reconheça o acidente de trabalho; Interação da equipe de saúde para o atendimento ao Acidente de Trabalho; Capacitação para o atendimento ao acidentado do trabalho; Preenchimento do formulário para a Notificação do Acidente de Trabalho (Ficha do SINAN).

No intuito de contribuir para aumento das notificações dos acidentes de trabalho, para a humanização do atendimento ao acidentado nas unidades básicas de saúde e nas emergências hospitalares e a garantia de seus direitos trabalhistas e previdenciários, a autora recomenda o efetivo compromisso dos gestores, na implantação do protocolo de atendimento ao acidente de trabalho, procedendo às orientações e os encaminhamentos aos órgãos de proteção e promoção 
da saúde do trabalhador. Recomenda também a assistência ao acidentado do trabalho por equipe multiprofissional, desenvolvendo ações interdisciplinares e intersetoriais. É importante ressaltar que a participação da sociedade, resultante do fortalecimento dos movimentos sociais no período de redemocratização do País, em especial dos sindicatos, e com a produção intelectual e científica sobre Saúde do Trabalhador e a sua disseminação para além do ambiente acadêmico. A documentação dessas ações para a avaliação do grau de implantação, oferta, utilização, cobertura e impacto ainda é, todavia, insuficiente. É extensa a sub-notificação dos agravos e doenças ocupacionais e relacionadas com o trabalho nos sistemas de informação do SUS, em que pesem os esforços para sua melhoria, o que vem ocorrendo gradualmente. $\mathrm{O}$ registro de doenças e agravos ocupacionais no SINAN é ainda pouco expressivo, considerando que representa um pequeno percentual dos registros da Previdência Social, que cobre menos da metade dos trabalhadores do País. Como o subregistro é disseminado, também os dados da Previdência Social são afetados. Este quadro é agravado pela pouca atribuição de nexo ocupacional/nexo com o trabalho nos diagnósticos clínicos, feitos pela perícia médica nos casos de incapacidade para o trabalho. (SERVO,2005)

Permanecem grandes dificuldades na consolidação e legitimação da Saúde do Trabalhador no SUS. Por exemplo, são muitas as dificuldades dos gestores, em particular, de reconhecer o trabalho como um dos determinantes do processo saúde/doença, ou a sua relevância para que se considerem as ações em Saúde do Trabalhador como prioridade de política. Há também um desconhecimento expressivo, por parte dos diferentes profissionais de saúde, dos agentes de riscos e agravos à saúde relacionados com o trabalho, e da existência de estratégias viáveis de prevenção ou de promoção da saúde do trabalhador com mudanças nos ambientes de trabalho.

O movimento sindical tem se tornado mais frágil diante da elevada proporção de desempregados e da reestruturação produtiva que vem impondo importantes mudanças no mundo do trabalho, com índices expressivos de trabalho informal. Por fim, em que pese à existência de algumas experiências exitosas em vários municípios do país, no plano nacional, existe uma insuficiente articulação entre as instituições responsáveis pela saúde, segurança e proteção social do trabalhador, e também com a atenção primária em saúde, tanto com o Programa Saúde da Família, como com o Programa de Agentes Comunitários de Saúde. ( SANTANA) No âmbito específico do SUS, os desafios da RENAST são muitos, mas um dos mais fundamentais é a formação, cada vez maior, de profissionais habilitados para enfrentarem os desafios que a prática interdisciplinar e intersetorial da Saúde do Trabalhador. A atividade produtiva domiciliar, o comércio em vias públicas e o trabalho no campo, por exemplo, certamente mobilizarão o SUS para uma integração com o PACS e o PSF, que em sua conhecida capilaridade poderão definir um novo patamar de ações de Saúde do Trabalhador articulada à atenção básica de saúde.( SANTANA). 


\section{REFERÊNCIAS BIBLIOGRÁFICAS}

1. BRASIL, Ministério da Saúde. PORTARIA № 777 /GM Em 28 de abril de 2004. Dispõe sobre os procedimentos técnicos para a notificação compulsória de agravos à saúde do trabalhador em rede de serviços sentinela específica, no Sistema Único de Saúde - SUS. Disponível em:< http://www.renastonline.org/node/70> acesso em 03 de abril de 2012.

2. BRASIL. Leis. Lei Orgânica da Saúde no $\mathbf{8 0 8 0}$, de 19 de setembro de 1990. Dispõe sobre as condições para promoção, proteção e recuperação da saúde, a organização e o funcionamento dos serviços correspondentes e dá outras providências. Diário Oficial da União, Brasília, 1990.

3. DIAS, E. C.; HOEFEL, M. G. ; O desafio de implementar as ações de saúde do trabalhador no SUS: a estratégia da RENAST. Disponível em:< http://www.scielo.br/pdf/csc/v10n4/a07v10n4.pdf > acesso em 20 agosto de 2012.

4. Gerência Regional de Saúde de Sete Lagoas (GRS- SL)- Notificações de Agravos em Saúde do Trabalhador no período de 2007 a 2010. Disponível em:<

http://www.saude.mg.gov.br/politicas_de_saude/visa/GRS\%20Sete\%20Lagoa s.pdf> acesso em 10 de setembro de 2012 .

5. MINISTÉRIO DA SAÚDE- SECRETARIA DE ATENÇÃO À SAÚDE. DEPARTAMENTO DE AÇÕES PROGRAMÁTICAS ESTRATÉGICAS ÁREA TÉCNICA DE SAÚDE DO (A) TRABALHADOR (A)- COSAT - POLÍTICA NACIONAL DE SAÚDE DO (a) TRABALHADOR (a). Brasília. Janeiro de 2004. Disponível em:

<http://portal.saude.gov.br/portal/arquivos/pdf/proposta_pnst_st_2009.pdf > acesso em 25 de setembro de 2012.

6. RENAST- ONLINE; Diretrizes de implantação da Vigilância em Saúde do Trabalhador no SUS. Disponível em: <

http://www.renastonline.org/recursos/diretrizes-de-implanta\%C3\%A7ao-da-vigil\%C3\%A2ncia-em-sa\%C3\%BAde-do-trabalhador-no-sus> Acesso em 08 de setembro de 2012.

7. REDE NACIONAL DE ATENÇÃO INTEGRAL À SAÚDE DO TRABALHADOR - MANUAL DE GESTÃO E GERENCIAMENTO capítulo 3 pág $37 p$ - 1를 edição - 2006 - Hemeroteca Sindical Brasileira - São Paulo, SP disponível em :< http://portal.saude.gov.br/portal/arquivos/pdf/ManualRenast07.pdf

8. SANTANA, V. S. ; SILVA, J.M.; Os 20 Anos da Saúde do Trabalhador no SUS: limites, avanços e desafios. Disponível em: < http://www.saude.mg.gov.br/politicas_de_saude/visa/Os\%2020\%20anos\%20d a\%20ST\%20no\%20SUS-\%20Limites,\%20avancos\%20e\%20desafios.pdf> Acesso em 08 de outubro de 2012.

9. SERVO, L. M. S. III SEMINÁRIO POLÍTICAS SOCIAIS E CIDADANIA.Reflexões teóricas sobre a importância da notificação de acidentes de trabalho para a saúde do trabalhador e previdência social. Disponível em : < 
http://www.interativadesignba.com.br/llI_SPSC/arquivos/sessao2/062.pdf> acesso em 22 de agosto de 2012.

10.TAMASSIRO,C.U.; MENDES,N.K.G.; MONTICELLI,R.G.M.; PASSALON,R.R., Atribuições da enfermagem na saúde do trabalhador. Recife- PE. 2009. Disponível em:< http://www.abeneventos.com.br/2senabs/cd_anais/pdf/id34r0.pdf> acesso em 25 de julho de 2012. 